\title{
Dynamics of Midlatitude Tropopause Height in an Idealized Model
}

\author{
PABLO ZURITA-GOTOR \\ Universidad Complutense and Instituto de Geociencia, Madrid, Spain \\ GEOFFREY K. VALLIS \\ GFDL, and Princeton University, Princeton, New Jersey
}

(Manuscript received 6 August 2010, in final form 23 November 2010)

\begin{abstract}
This paper investigates the factors that determine the equilibrium state, and in particular the height and structure of the tropopause, in an idealized primitive equation model forced by Newtonian cooling in which the eddies can determine their own depth. Previous work has suggested that the midlatitude tropopause height may be understood as the intersection between a radiative and a dynamical constraint. The dynamical constraint relates to the lateral transfer of energy, which in midlatitudes is largely effected by baroclinic eddies, and its representation in terms of mean-flow properties. Various theories have been proposed and investigated for the representation of the eddy transport in terms of the mean flow, including a number of diffusive closures and the notion that the flow evolves to a state marginally supercritical to baroclinic instability. The radiative constraint expresses conservation of energy and so must be satisfied, although it need not necessarily be useful in providing a tight constraint on tropopause height.

This paper explores whether and how the marginal criticality and radiative constraints work together to produce an equilibrated flow and a tropopause that is internal to the fluid. The paper investigates whether these two constraints are consistent with simulated variations in the tropopause height and in the mean state when the external parameters of an idealized primitive equation model are changed. It is found that when the vertical redistribution of heat is important, the radiative constraint tightly constrains the tropopause height and prevents an adjustment to marginal criticality. In contrast, when the stratification adjustment is small, the radiative constraint is only loosely satisfied and there is a tendency for the flow to adjust to marginal criticality. In those cases an alternative dynamical constraint would be needed in order to close the problem and determine the eddy transport and tropopause height in terms of forcing and mean flow.
\end{abstract}

\section{Introduction}

Although the tropopause is one of the most important features of the atmospheric circulation, our understanding of the processes that control its height remains incomplete. Indeed, there is no unique definition of the tropopause. Conceptually, the tropopause may be thought of as the transition region separating the dynamically active troposphere, a layer in which mixing by the air motion takes place on time scales of days, and a stratosphere that is much more quiescent in comparison and so has a temperature closer to radiative equilibrium. Most physically based definitions of the tropopause hinge on this distinction,

Corresponding author address: Pablo Zurita-Gotor, Departamento de Geofísica y Meteorología, Universidad Complutense, Facultad de Ciencias Físicas, Madrid 28040, Spain.

E-mail: pzurita@alum.mit.edu whether the tropopause is defined in terms of the kinematic properties of the flow, the change in the air masses [particularly tracer concentration, including potential vorticity (PV)] across the tropopause, or the dynamical transport of entropy. An alternative definition of tropopause is given by the World Meteorological Organization's (WMO's) thermal definition as the level where the lapse rate first reaches (and stays above) $2 \mathrm{~K} \mathrm{~km}^{-1}$ (WMO 1957; Lewis 1991). However, note that unlike tropopause definitions based on mixing, there is nothing fundamental about this definition; it is best regarded as a consequence of the heating structure for the present-day atmosphere rather than as a defining property of the tropopause. Thus, there should be no expectation for this definition to retain its meaning in different climates. However, note that even if a dynamical definition of the tropopause is to be preferred, this does not mean that diabatic effects are not important. 
In the extratropics the bulk of the meridional and vertical transport of entropy is performed by the baroclinic eddies, so the question of what determines the tropopause height is essentially equivalent to the question of what determines the vertical scale of the eddies. This is a very challenging question, lying at the core of the wave-mean flow equilibration problem and also involving, as noted above, diabatic effects. Linear theory and dimensional analysis provides us with some guidance at least in the adiabatic problem (Held 1978; Branscome 1983). In the simplest case of the Charney-Boussinesq problem (uniform shear and stratification for a Boussinesq fluid on a beta plane), there is only one vertical scale in the problem, the Charney height, given by

$$
h=-\frac{f}{\beta} \frac{\partial_{y} \bar{\theta}}{\partial_{z} \bar{\theta}},
$$

and the vertical scale of the most unstable mode should scale with this height. Assuming that this defines the tropopause height $H$, then the "criticality parameter"

$$
\xi \equiv-\frac{f}{\beta H} \frac{\partial_{y} \bar{\theta}}{\partial_{z} \bar{\theta}}=\frac{h}{H}
$$

is unity by definition. This parameter is similar to the one determining whether a zonal flow is stable in the linear two-layer quasigeostrophic model (e.g., Vallis 2006), but it is not necessarily a good measure of baroclinic instability: that may occur with $\xi<1$, especially in inhomogeneous flows. Nevertheless, the parameter $\xi$ may determine the scale of the nonlinear flow. This is because it may be shown that the Rossby radius $(N H / f)$ scales with the Rhines scale $\left(\sqrt{u_{\mathrm{rms}} / \beta}\right)$ if the criticality is unity. That is, the scale of linear instability is sufficiently large that there is no room for an inverse cascade if the latter is halted at the Rhines scale (Held and Larichev 1996; Schneider and Walker 2006; Vallis 2006).

How relevant are these considerations for the forceddissipative equilibration problem? Historically, two different closures have been suggested for this problem: baroclinic adjustment and various forms of turbulent diffusion. Baroclinic adjustment assumes the existence of some preferred equilibrium state(s), usually the above condition: $\xi=1$. When the heating varies, this requires that the eddy fluxes adjust to balance the heating, which essentially makes the fluxes a function of the heating rather than a function of the mean state alone. Although this approach was originally motivated by the notion of baroclinic neutrality (Stone 1978; Lindzen 1993), similar conclusions may be obtained using other arguments not relying on baroclinic neutrality, which may be termed "marginal criticality" to distinguish them (Schneider 2004). (For definiteness we will henceforth refer to all such classes of arguments as marginal criticality, but without any implication as to what the mechanism or justification for such a process is.) In contrast, diffusive closures usually assume that the fluxes vary continuously with the mean state gradient, with a diffusivity that is in general also a function of the mean state (e.g., Green 1970; Stone 1972). For both closures to be compatible, the diffusivity or the heating would have to adjust in some particular way, as discussed more below. (Of course, without a prediction of the diffusivity a diffusive closure is rather empty of content.)

When $\xi \approx 1$, we have $(f / \beta)\left|\partial_{y} \bar{\theta}\right| \approx H \partial_{z} \bar{\theta}$, implying that the characteristic isentrope spans a meridional distance on the order of the planet size $f / \beta \sim a$ as it rises from the surface to the tropopause. Thus, the typical air parcel warming up at the subtropical boundary layer finds its cooling level around the polar tropopause (Fig. 1a). Now, if the eddies are quasi-adiabatic the isentropic slope agrees with the mixing slope (Zurita-Gotor and Vallis 2009), so this can only occur if the ratio between meridional and vertical heat transport satisfies

$$
\frac{Q_{H}}{Q_{V}} \sim \frac{\overline{v^{\prime} \theta^{\prime}}}{\overline{w^{\prime} \theta^{\prime}}} \sim-\frac{\partial_{z} \bar{\theta}}{\partial_{y} \bar{\theta}} \sim \frac{f}{\beta H},
$$

where $Q_{H}$ and $Q_{V}$ are the horizontal and vertical heating [see Zurita-Gotor and Vallis (2010) for specific definitions]. Hence, for $\xi \approx 1$ the heating cannot be arbitrary but must adjust to satisfy this special condition. Assuming that the heat fluxes are independently determined by a diffusive closure (Zurita-Gotor and Vallis 2009), this condition then becomes a theory for tropopause height. For instance, consider the scenario depicted in Fig. 1b, in which the $\overline{w^{\prime} \theta^{\prime}}$ to $\overline{v^{\prime} \theta^{\prime}}$ ratio leads to an isentropic slope as depicted by the thick line. To satisfy the condition $\xi \approx 1$, this line should be extended to the poleward region, where air parcels would cool at a tropopause level $H=h$. However, this may not be possible if the vertical extent of the heating is constrained by the heating formulation. For instance, if the formulation were such that the heating could only extend to a height $H_{\text {rad }}<h$, then the flow would be supercritical $(\xi>1)$ unless the isentropes adjust to the new slope indicated by the thin line. This would require that the diffusivity adjusts to produce the appropriate transport, instead of being a predetermined function of the mean state.

We can see that an important difference between the actual forced-dissipative problem and the linear problem is that for the former diabatic considerations may play a role and the vertical scale of the eddies is not 
a

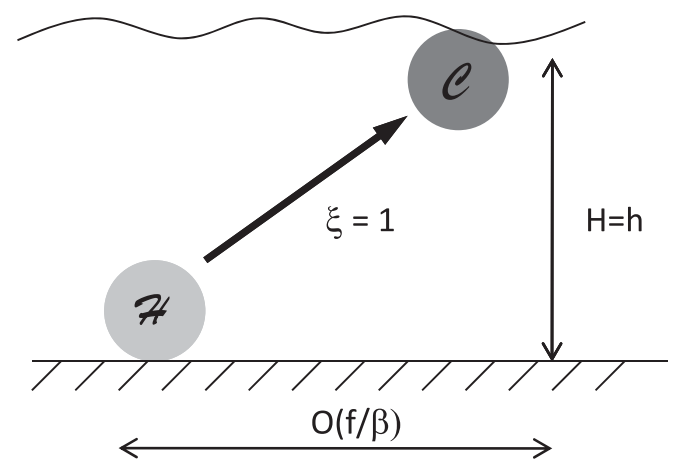

b

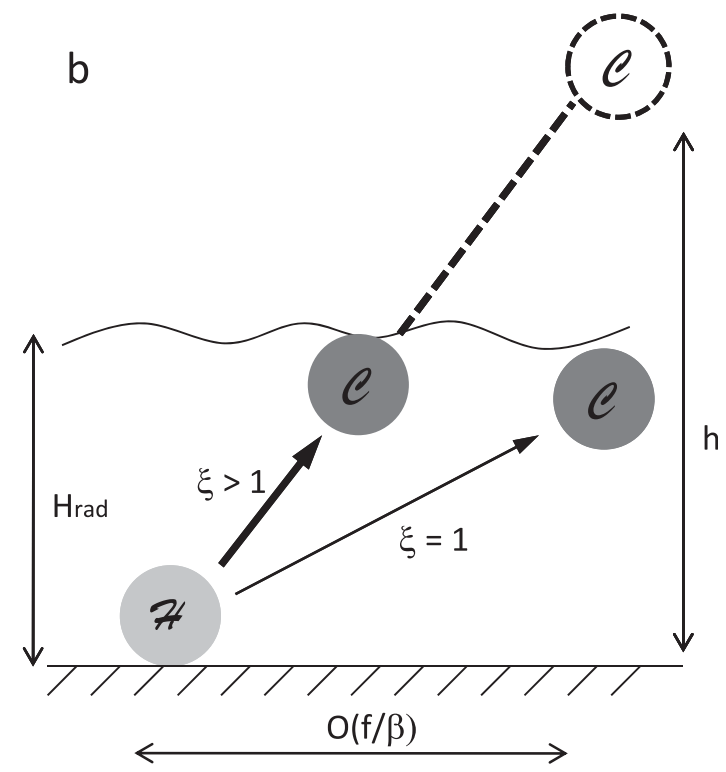

FIG. 1. (a) The characteristic isentropic slope when $\xi \approx 1$; (b) the isentropic slope (thick line) when the vertical scale of the heating limits the tropopause height $\left(H_{\mathrm{rad}}<h\right)$. In that case, the flow is supercritical $(\xi>1)$ unless the isentropic slope flattens to the thin line.

necessarily determined by the dynamics alone. One may think of the heating formulation as introducing additional vertical scale(s), which could compete with $h$ for determining the tropopause height. The role of the diabatic processes for constraining the tropopause height was noted by Held (1982), who introduced the notion of a radiative constraint. The basic idea is as follows: if the vertical mixing by the dynamics produces some tropospheric stratification $\widetilde{\theta}_{z}$ up to the tropopause $H$, with the fluid remaining in radiative equilibrium aloft, then $H$ and $\widetilde{\theta}_{z}$ cannot be arbitrary because the net column heating must remain unchanged when heat is redistributed vertically. Thus, for any given value of $\widetilde{\theta}_{z}$ there is only one value of $H$ producing the right outgoing longwave radiation at the top of the atmosphere, and vice versa. The ensuing relation between $H$ and $\widetilde{\theta}_{z}$ is called the radiative constraint.

To close the problem, an additional dynamical constraint between $H$ and $\widetilde{\theta}_{z}$ is needed, and the baroclinic adjustment/marginal criticality condition $\xi=1$ is one such. However, if this constraint does hold, then a diffusive constraint with predetermined diffusivity cannot simultaneously be valid because the system is overdetermined, for reasons discussed above. The question then becomes whether turbulent diffusion or baroclinic adjustment, or some other theory, is more relevant for the extratropical equilibrium, and if and how the radiative constraint is effective.

Previous work has not been conclusive on this issue. Thus, Thuburn and Craig (1997) used a comprehensive
GCM to test various theories for the height of the tropopause, finding that although the radiative constraint was a useful perspective, dynamical constraints based on theories of baroclinic adjustment were not in general satisfied. In contrast, Schneider and Walker (2006) found robust adjustment to marginal criticality in an idealized dry GCM forced by Newtonian cooling, though they did not test the role of radiative constraint explicitly. Other studies with different heating formulations have not found adjustment to marginal criticality. For instance, gray radiation models tend to produce less stratified, more supercritical mean states (Frierson et al. 2006), while significant changes in criticality can be found when using a different time scale for the eddies and the mean in a Newtonian model (Zurita-Gotor 2008). Models with explicit moist effects exhibit sensitivity of the criticality on moisture (Schneider and O'Gorman 2008). In a more idealized framework, Zurita-Gotor (2007) found that the criticality varied slowly but smoothly with the forcing in a forced-dissipative, two-layer quasigeostrophic model, suggesting that the system had no preferred equilibria. Zurita-Gotor and Vallis (2009) found similar results for a two-level primitive equation model when the stratification (internally determined now) was diagnosed from the model. Finally, Zurita-Gotor and Vallis (2010) studied the implications of the diffusive closure for the sensitivity of the mean state on the heating. However, in these three studies the depth of the mixing is virtually prescribed by construction, which is unavoidable for the two-level system. 
The disparity of results when different heating formulations are used suggests that the relevance of the different constraints might be model or parameter dependent. For instance, it was argued above that the adjustment to marginal criticality is to be expected in the unforced Charney-Boussinesq problem, in which the only dimensional height entering the problem is the Charney height and there is no radiative constraint. In the opposite limit, one might conceive a heating formulation in which the radiative constraint $H=f\left(\widetilde{\theta}_{z}\right)$ was so flat that it would not allow the tropopause height to depart much from some value $H_{\text {rad }}$, which would be akin to a rigid lid. In this limit in which the vertical eddy scale is externally constrained, we might expect to observe supercriticality as in the two-level study of Zurita-Gotor and Vallis (2009), at least for sufficiently strong forcing. More generally, the radiative and marginal criticality constraints together fully determine the vertical temperature structure with no room for a diffusive closure. Conversely, if a diffusive closure with predetermined diffusivity holds, then the marginal criticality and radiative constraints cannot be simultaneously satisfied.

One may therefore envision several scenarios. For example, the radiative constraint could play the role of the rigid lid in limiting the vertical expansion of the eddies, allowing supercritical flow even when the tropopause height is not absolutely fixed. It is also possible that some of the assumptions behind the application of the radiative constraint (uniform tropospheric stratification and stratosphere in radiative equilibrium) are too idealized for this constraint to be useful. Thus, although the radiative constraint, as a statement of energy conservation, must always be satisfied, in practice it could be ineffective at constraining the mean state and preventing the adjustment to marginal criticality or to marginal supercriticality operating simultaneously with a diffusive mechanism. (The extent to which the radiative constraint can prevent the adjustment to marginal criticality is likely dependent on the heating formulation used and the parameter regime of interest.) Another possibility is that the diffusive mechanism is not particularly relevant for the extratropical equilibrium.

The goal of the present work is to clarify these possibilities and to try to understand the interaction of the radiative and dynamical constraints, and to introduce some objective or quantitative parameters to characterize their influence. The work may be considered an extension of the previous work of the authors to the more realistic case of a continuous fluid in which the vertical scale of the mixing is internally determined, while trying to maintain a relatively idealized framework to aid physical interpretation. Specifically, this paper investigates whether the radiative and marginal criticality constraints can predict the sensitivity of tropopause height in an idealized model when the external parameters are varied, or whether some other dynamical constraint is needed.

The paper is structured as follows. Section 2 describes the formulation and numerics of the specific numerical model that we use. Section 3 presents the radiative constraint for this model and discusses its properties. Section 4 describes the sensitivity of the tropopause in our runs when the main external parameters are varied, focusing on the agreement with the different predictions. Finally, section 5 concludes with some discussion of our results and a brief summary.

\section{Model description}

We use for this study the Massachusetts Institute of Technology (MIT) GCM (Marshall et al. 1997; see http://mitgcm.org/), a multilevel primitive equation model well suited to idealized modeling because of its flexible configuration. We use a hydrostatic, Boussinesq, betaplane version of the model, with a linear equation of state with thermal expansion coefficient $\alpha_{T}=3.33 \times 10^{-3} \mathrm{~K}^{-1}$ (obtained linearizing the ideal gas law; see Zurita-Gotor and Vallis 2009), configured in a channel with slippery zonal walls. By using a Boussinesq model we lose some realism vis-à-vis the real atmosphere, but we are able to focus more on the dynamical mechanisms because there is no longer a vertical scale height for density. We added to this model simple forcing functions similar to those described by Held and Suarez (1994). The horizontal wind is damped linearly over a boundary layer with fixed depth $\left(h_{\mathrm{BL}}=3 \mathrm{~km}\right)$, with a damping rate that is maximum at the surface $\left(\tau_{F}^{-1}\right)$ and decreases linearly to zero over that height. The thermal field is forced by Newtonian relaxation with time scale $\tau$ to a prescribed "radiative equilibrium" profile of the form

$$
\begin{aligned}
\theta_{R}(y, z)= & \theta_{0}+\int_{0}^{z}\left[\theta_{z \infty}+\left(\theta_{z 0}-\theta_{z \infty}\right) e^{-z^{\prime} / H_{R}}\right] d z^{\prime} \\
& -\frac{\delta_{Y}}{2} \tanh \left[\frac{y-\left(L_{Y} / 2\right)}{\sigma}\right] e^{-z / H_{Y}}
\end{aligned}
$$

In this profile, the stratification transitions from its surface value $\theta_{z 0}$ to a value $\theta_{z_{\infty}}$ at large heights, with the transition occurring over a depth of order $H_{R}$. Additionally, the radiative equilibrium baroclinicity decays vertically over a depth $H_{Y}$, which we introduced mainly for numerical convenience. We chose a large enough value that the zonal wind only departs from the constant shear profile at heights well above the simulated tropopause. At the same time, by using a finite $H_{Y}$ we reduce the vertical shear and maximum zonal wind near 
the top of a domain that extends much farther than the simulated tropopause, easing the stability constraints on the time step. Note that this profile produces a monotonic PV gradient with no interior extrema, so there is not a "radiative-equilibrium tropopause."

For our control run we take $\theta_{z 0}=0, \theta_{z_{\infty}}=20 \mathrm{~K} \mathrm{~km}^{-1}$, $H_{R}=10 \mathrm{~km}, H_{Y}=50 \mathrm{~km}, f_{0}=10^{-4} \mathrm{~s}^{-1}, \beta=1.6 \times$ $10^{-11} \mathrm{~m}^{-1} \mathrm{~s}^{-1}, \delta_{y}=60 \mathrm{k}, L_{y}=12500 \mathrm{~km}, \sigma=1000 \mathrm{~km}$, a frictional time scale $\tau_{F}=1$ day, a Newtonian time scale $\tau=20$ days, and biharmonic diffusion with coefficient $\nu=10^{16} \mathrm{~m}^{4} \mathrm{~s}^{-1}$ in the momentum equations. We use a convective adjustment scheme for the runs with negative $\theta_{z 0}$ but the results were insensitive when some of these simulations were repeated using grid-scale convection only. The channel length is $L_{X}=32000 \mathrm{~km}$ and the computational model top is set at a height $H_{T}=$ $40 \mathrm{~km}$. We use a uniform $128 \times 48$ horizontal grid, and a nonuniform vertical grid with 80 grid points. The vertical spacing is $250 \mathrm{~m}$ over the lowest $15 \mathrm{~km}$ of the domain, increasing gradually above that level to a maximum grid size of $2200 \mathrm{~m}$. We have also introduced a sponge layer over the upper $10 \mathrm{~km}$ of the domain to prevent the buildup of eddy activity near the top of the domain (for which we use a free surface boundary condition) and unphysical downward reflection. The sponge damps $e d d y$ momentum ( $u^{\prime}$ and $v^{\prime}$ ) only, and its damping rate decays quadratically to 0 from a maximum value of $(3 \mathrm{~h})^{-1}$ at the top. We performed some additional simulations in a domain twice as deep for the control run and for two other runs with parameter configurations conducive to high tropopauses $\left(f_{0}=5 \times 10^{-4} \mathrm{~s}^{-1}\right.$ and $\theta_{z 0}=-30 \mathrm{~K} \mathrm{~km}^{-1}$ ) to ensure that the results are insensitive to $H_{T}$. The diagnostics presented are averages from the last 300 days in 600-day simulations starting from radiative equilibrium.

Figure 2 describes the mean state for our control run, which uses a radiative-equilibrium profile that is convectively neutral at the surface $\left(\theta_{z 0}=0\right)$. This leads to a fairly well-stratified mean state as shown in Fig. 2a (see also Fig. 5d). The different mean and eddy fields have realistic structure and values, including the characteristic three-cell structure in the mean meridional circulation. We define the troposphere as the boundary layer over which the bulk of the dynamical adjustment and transport occurs and estimate the tropopause as the height over which the isentropic mass flux decreases to a $30 \%$ of its maximum value at each latitude. We chose this conservative value motivated by the large variability among runs in the fraction of eddy activity that leaks into the stratosphere. Although the isentropic mass flux does not drop as fast in our Boussinesq fluid as in the compressible atmosphere, the decay is still sharp enough that the sensitivity of tropopause height is very similar when using a smaller threshold. Note that this tropopause (depicted with a thick line in Fig. 2) is very different from what one would obtain using the WMO tropopause definition, given the very different heating structure in our model compared to the real atmosphere. In fact, the WMO definition is not very meaningful for our model, in which temperature always increases with height (temperature and potential temperature are equivalent for a Boussinesq fluid). The tropopause may not even show up clearly in the thermal structure in all cases (cf. Figs. 5c,d): it only does so when the dynamically adjusted stratification is significantly different from its radiative-equilibrium counterpart at tropopause levels.

\section{The radiative constraint}

One way to think about the radiative constraint of Held (1982) is to first suppose that the horizontal heat transport problem is solved and to focus on the vertical heat transport. Because the net column heating is then known, the vertical thermal structure cannot be arbitrary but is constrained by the requirement that the column diabatic cooling balances the prescribed external heating. In the simplest situation in which the vertical thermal structure is idealized as consisting of a stratosphere in strict radiative equilibrium lying above a uniformly stratified troposphere, the thermal structure is fully determined by just two parameters: the tropospheric stratification and tropopause height. The constraint on the column heating then implies that these two parameters must be related. We refer to this relation as the idealized radiative constraint, to emphasize the fact that this constraint only holds when the simple idealization of the thermal structure put forward above is appropriate. (The radiative constraint itself, as a statement of conservation of energy, must always hold.)

Figure 3 illustrates the application of this idea with a Newtonian cooling model. The thin line shows a typical radiative-equilibrium profile toward which the flow is relaxed, convectively unstable in the lower troposphere and very stably stratified in the stratosphere, with the transition occurring around some height $H_{R}$. The thick line shows the adjusted profile after the dynamical transport, which we assume to be uniformly stratified. The area between the curves determines the net column heating. Since this heating has to be consistent with the meridional transport problem, only one stratification is possible if the tropopause height is given. For instance, at the latitude of maximum meridional heat transport (or in a horizontal average), the vertically integrated dynamical heating $\left(\partial_{y} \int \overline{v \theta} d z\right)$ vanishes and the adjusted stratification must be such that the two lobes have equal area: 

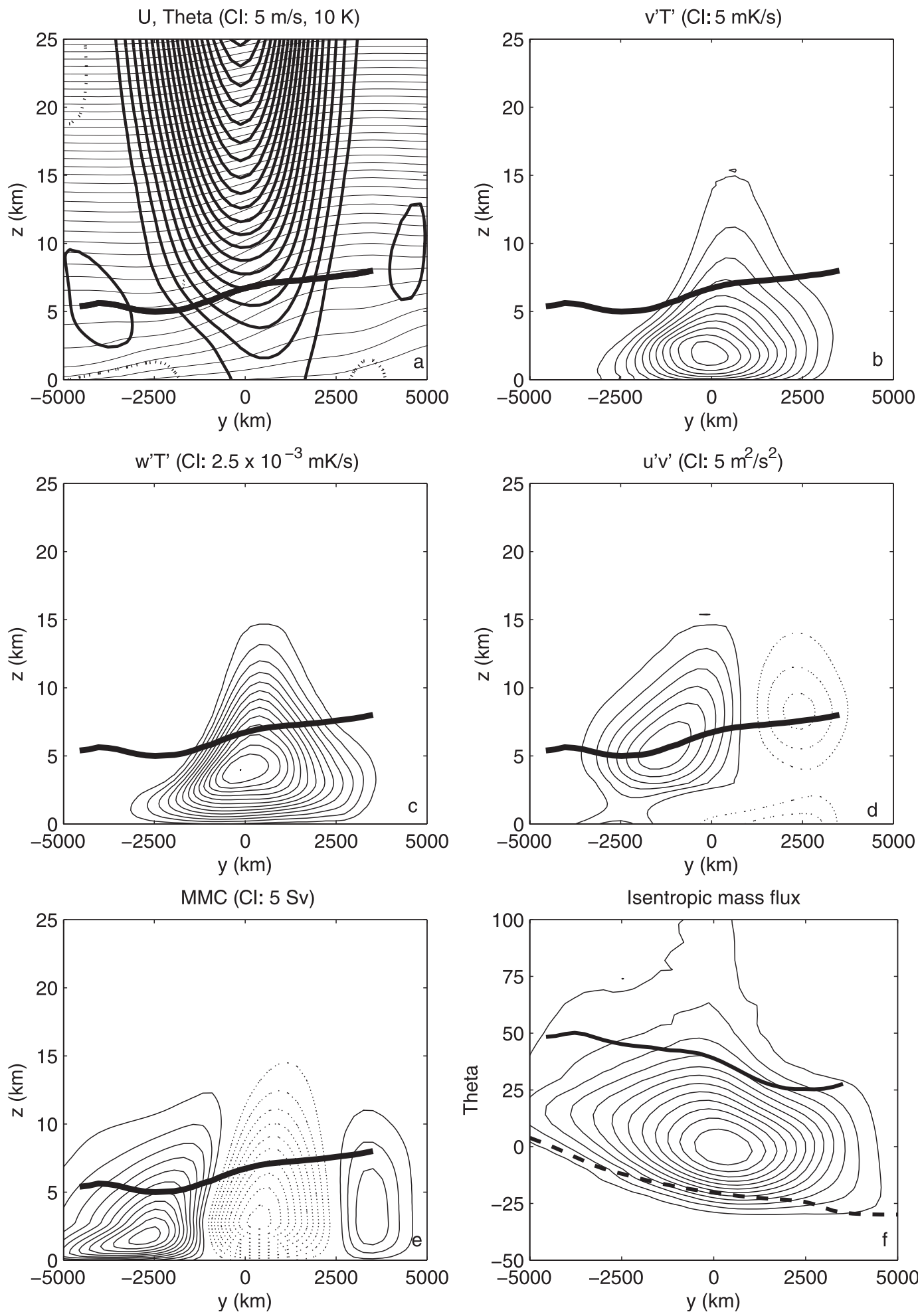

FIG. 2. Description of the equilibrium state for the control run. (a) Mean zonal wind (thick) and potential temperature (thin); (b) meridional eddy heat flux; (c) vertical eddy heat flux; (d) eddy momentum flux; (e) mean meridional circulation mass streamfunction; (f) isentropic mass flux (and mean lowest-level temperature). Contour interval $(\mathrm{CI})$ is indicated in each panel title and the tropopause is shown with a thick line. 


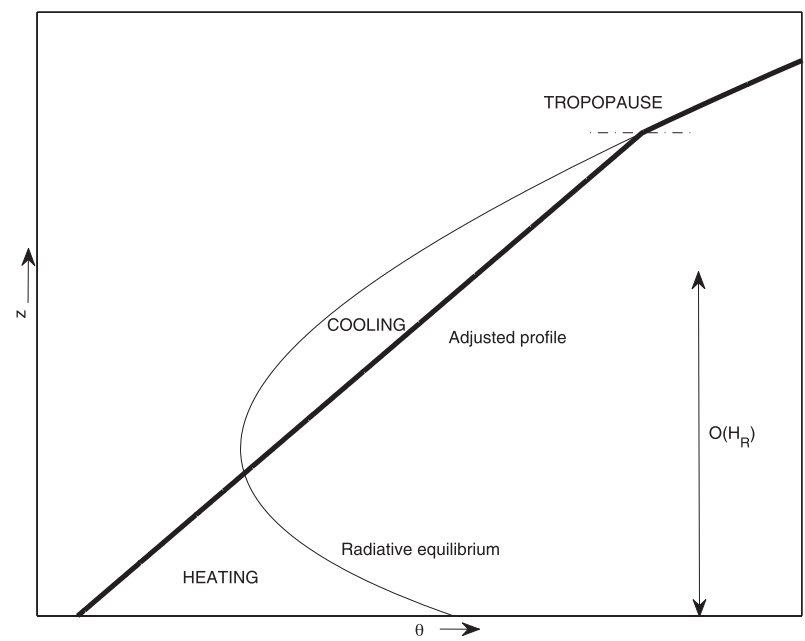

FIG. 3. Sketch illustrating the application of the radiative constraint at the latitude of maximum meridional heat transport. At that latitude, the net heat content of the column is unchanged from radiative equilibrium and the areas of cooling and heating are equal.

$$
\begin{aligned}
\int_{0}^{H}\left[\theta_{A}(z)-\theta_{R}(z)\right] d z \\
\quad=\int_{0}^{H}\left[\theta_{R}(H)-(H-z) \tilde{\theta}_{z}-\theta_{R}(z)\right] d z=0,
\end{aligned}
$$

where $\theta_{A}(z)$ is the adjusted profile, with uniform stratification $\tilde{\theta}_{z}, \theta_{R}(z)$ is the radiative-equilibrium profile, and $H$ is the tropopause height. Note that there is no distinction between temperature and potential temperature in our Boussinesq fluid, so the assumptions of a uniform stratification or a uniform lapse rate are equivalent.

For the radiative-equilibrium profile $\theta_{R}$ considered in this paper, namely (4), the radiative constraint can be solved analytically to produce the following relation between $H$ and $\tilde{\theta}_{z}$ :

$$
\frac{\theta_{z^{\infty}}-\widetilde{\theta}_{z}}{\theta_{z_{\infty}}-\theta_{z 0}}=2 \frac{1-(1+\eta) e^{-\eta}}{\eta^{2}}
$$

where $\eta=H / H_{R}$. This relation, plotted in Fig. 4a, predicts the rising of the tropopause as the stratification $\tilde{\theta}_{z}$ increases (for fixed $\theta_{z 0}, \theta_{z \infty}$ ), very steeply when the stratification is large. The normalization implies that for small $\theta_{z_{\infty}}-\theta_{z 0}$ the radiative constraint becomes "loose," in the sense that small changes in the stratification are associated with large changes in tropopause height. Equation (6) becomes singular in the pure Charney-Boussinesq limit $\theta_{z 0}=\theta_{z^{\infty}}$, a limit in which the scale of the eddies is no longer constrained by $H_{R}$.
It is also useful to define the steepness of tropopause height

$$
s_{H}=\frac{\partial \log H}{\partial \log \widetilde{\theta}_{z}},
$$

measuring the local sensitivity of tropopause height (as predicted by the idealized radiative constraint) to changes in stratification. A large steepness implies that $H$ is very sensitive to changes in the stratification, while small values of $s_{H}$ are associated with flat tropopause heights. Figure $4 \mathrm{~b}$ shows that for $\theta_{z 0} / \theta_{z^{\infty}} \rightarrow-\infty, s_{H} \rightarrow 0$ and we recover the rigid-lid case. In the opposite limit, $s_{H}$ becomes infinitely large (only some contours are shown) as we approach $\widetilde{\theta}_{z}=\theta_{z 0}$ or $\widetilde{\theta}_{z}=\theta_{z \infty}$. This represents a "freefloating" tropopause limit, in which the tropopause height is no longer constrained by stratification. In that limit, we expect the radiative constraint to play only a minor role in determining the height of the tropopause.

\section{Tightness of the radiative constraint}

As a statement of energy conservation, the radiative constraint must always be satisfied. Yet because some of the underlying assumptions behind the idealized radiative constraint (the construction in Fig. 3) may seem contrived, it is fair to try to determine the extent to which this constraint really does constrain the flow. For instance, the equal-area construction is sensitive to the vertical structure of the stratification: when this structure changes it is not sufficient to characterize the tropospheric stratification in terms of its mean value alone. The assumption of a stratosphere in strict radiative equilibrium is also an idealization because long waves penetrate across the tropopause and the stratospheric heating that they induce may have a significant impact on tropopause height (Thuburn and Craig 2000; Birner 2010). In the real world, both factors represent additional heating sources that may render the idealized radiative constraint construction inaccurate. Most importantly, if these heating sources are comparable to the dynamical heating then we would not necessarily expect the idealized radiative constraint to predict the correct sensitivity of tropopause height. In that case, changes in the stratospheric heating resulting from anomalous wave propagation across the tropopause and/or changes in the tropospheric heating due to changes in the vertical structure of stratification may dominate the changes in the dynamical heating when the mean tropospheric stratification changes.

To get a feeling for the relevance of this constraint in our model, Fig. 5 compares results for two different simulations. We first consider a simulation with statically unstable radiative-equilibrium stratification at the surface, $\theta_{z 0}=-20 \mathrm{~K} \mathrm{~km}^{-1}$. Figures $5 \mathrm{a}$ and $5 \mathrm{~b}$ show that the 


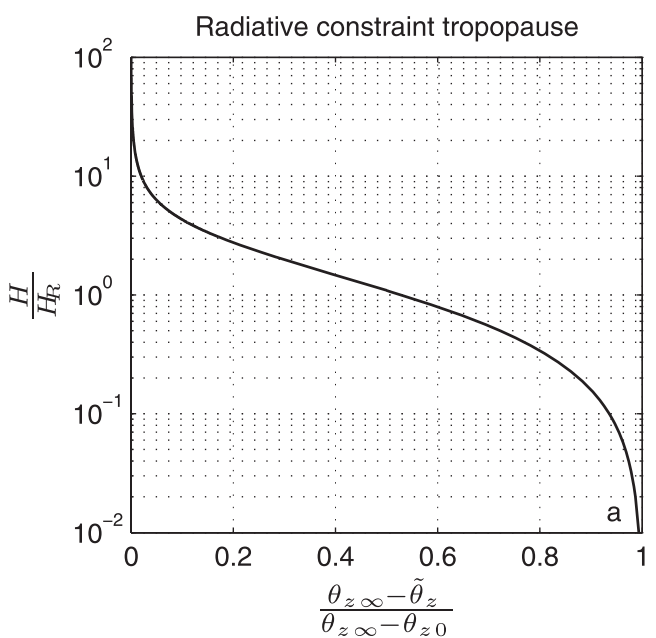

Tightness of radiative constraint

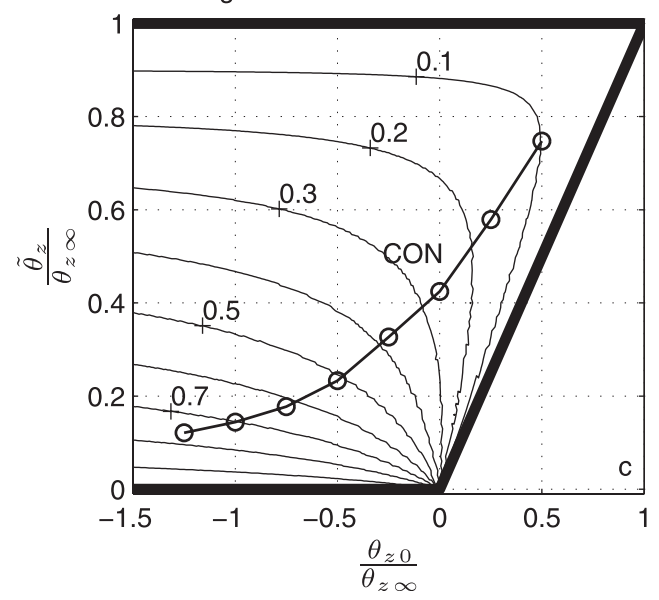

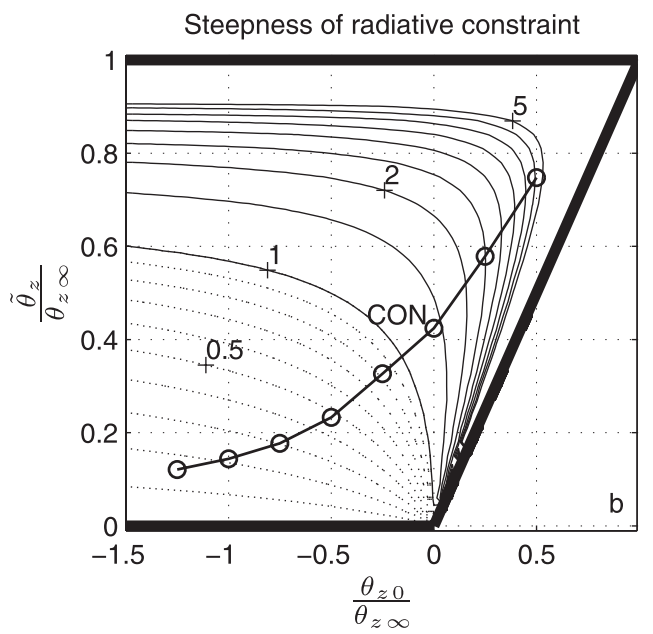

Sensitivity to $\theta_{z 0}$

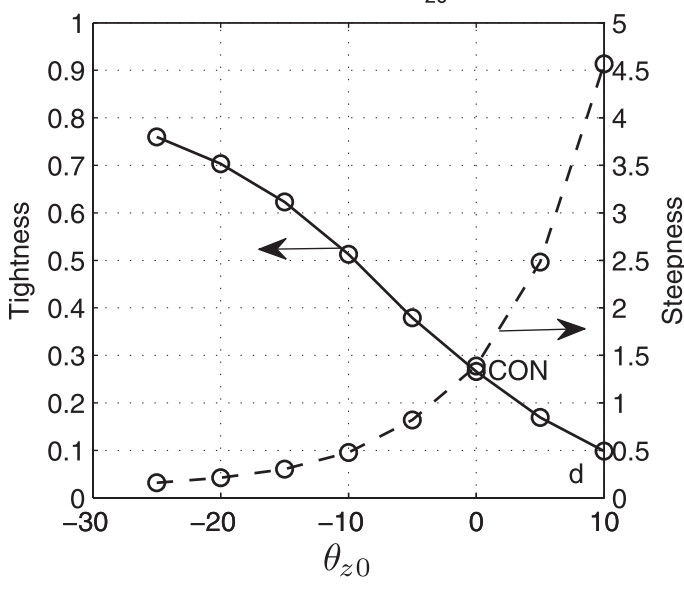

FIG. 4. Radiative constraint for the Newtonian cooling model: (a) tropopause height, (b) steepness of tropopause height ( $\mathrm{CI}$ is 0.1 for dashed contours and 0.5 for solid contours), (c) tightness of the radiative constraint ( $\mathrm{CI}$ is 0.1 ), and (d) tightness (solid, left axis) and steepness (dashed, right axis) for the equilibrated simulations varying $\theta_{z 0}$. These simulations are also mapped in (b) and (c). CON refers to the control simulation.

assumptions behind the idealized radiative constraint are clearly violated for this run. The stratification has vertical structure, the most dominant feature being a highly stratified surface layer presumably forced by the cold surface return flow (Held and Schneider 1999). Additionally, the large Eliassen-Palm flux across the tropopause in Fig. 5b implies that the stratosphere cannot be in radiative equilibrium. Although both features are apparent in the radiative constraint construction shown in Fig. 5c, this device still works because these corrections are much smaller than the dynamical adjustment in stratification implied by the equilibrium profile. In contrast, Fig. $5 \mathrm{~d}$ shows the same construction for our control run, which is only neutrally stratified at the surface in radiative equilibrium. In this case the time-mean stratification departs relatively little from its radiative-equilibrium profile and the corrections to the stratification implied by the violation of the assumptions behind the idealized radiative constraint are as important as the dynamical adjustment itself. Hence we do not expect this constraint to constrain the equilibrium state as much for this simulation as for the previous one. Generally speaking, we would expect the radiative constraint to work better when the dynamical vertical heat transport is large, which is typically associated with strong destabilization at the surface.

To be more quantitative about the effectiveness of the radiative constraint we define a tightness parameter $T$ :

$$
T \equiv 1-\frac{\widetilde{\theta}_{z}}{\theta_{z R}(H)},
$$

where $\theta_{z R}(H)$ is the radiative-equilibrium stratification at the tropopause. This parameter is small when the mean stratification in the upper troposphere does not 

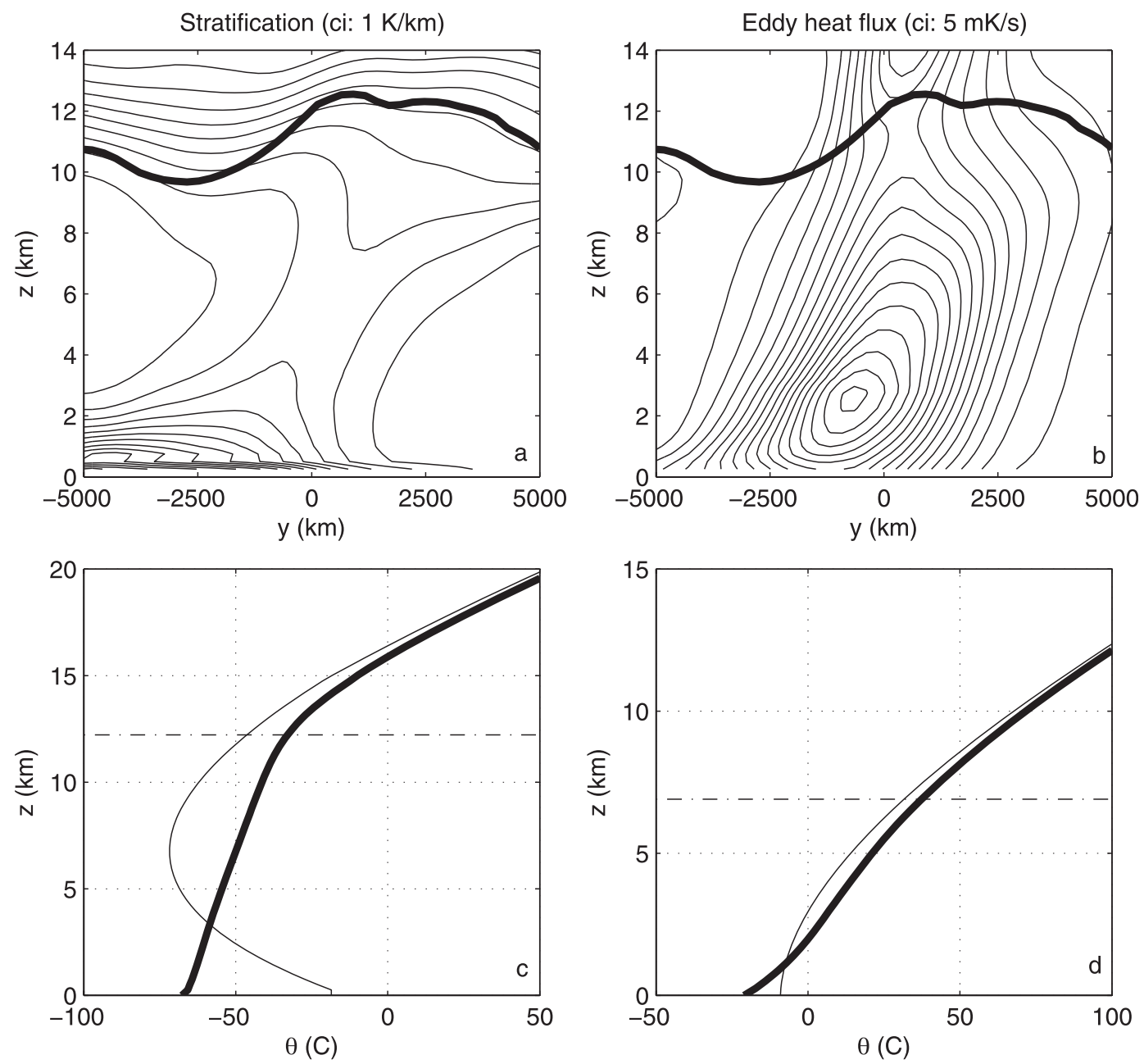

FIG. 5. For a run with $\theta_{z 0}=-20 \mathrm{~K} \mathrm{~km}^{-1}$, (a) stratification, (b) eddy meridional heat flux, and (c) radiative constraint construction (radiative-equilibrium temperature is thin and time-mean temperature thick). (d) As in (c), but for the control run, which has neutral radiative-equilibrium stratification at the surface. The tropopause is shown with thick lines in (a) and (b) and with dashed-dotted lines in (c) and (d).

depart much from its radiative-equilibrium value, while it approaches 1 when the stratification adjustment is significant. Hence, $T$ measures the importance of the vertical heat transport for the mean state, suggesting that the radiative constraint should be more effective as $T$ approaches 1 . Note that it is also only when $T$ is sufficiently close to 1 that the tropopause height becomes apparent in the thermal structure in the form of a discontinuity in the stratification (for reference, the simulation in Fig. $5 \mathrm{c}$ has $T=0.71$, whereas that in Fig. $5 \mathrm{~d}$ has $T=0.27)$. In the presence of a rigid lid at $z=H$ we may define $\theta_{z R}\left(H^{+}\right)=\infty$ to make $T=1$ in that limit.

Figure $4 \mathrm{c}$ shows the tightness of the radiative constraint in our model as a function of the different parameters. Comparing with Fig. 4b, it is apparent that $T$ and $s_{H}$ have very similar structure, so that the radiative constraint is tight (loose) when $s_{H}$ is small (large). In other words, the radiative constraint is more effective constraining the flow in the limit in which it does not let the tropopause move much. This is easy to understand from the graphical construction in Fig. 3 because when $T$ is small the radiativeequilibrium and adjusted profile are nearly parallel at tropopause levels, so that large changes in tropopause height (i.e., a large $s_{H}$ ) are associated with small changes in the upper tropospheric cooling (the area of the top lobe). To summarize, $T$ increases and $s_{H}$ decreases when $\theta_{z 0} / \theta_{z_{\infty}}$ decreases, reaching $T=1$ in the rigid-lid limit $\left(\theta_{z 0} / \theta_{z_{\infty}} \rightarrow-\infty\right)$ and $T=0$ in the uniform Charney limit of a free-floating tropopause $\left(\widetilde{\theta}_{z}=\theta_{z 0}\right.$ or $\left.\widetilde{\theta}_{z}=\theta_{z^{\infty}}\right)$.

\section{Tropopause sensitivity}

We have performed a large number of simulations varying the external parameters in our model, affecting 
the tropopause height. In this section we describe the sensitivity of tropopause height to the main parameters and use this sensitivity to test the usefulness and relevance of the marginal criticality and radiative constraints. The parameters that we have changed are the radiativeequilibrium baroclinicity $\delta_{Y}$, the radiative-equilibrium stratification at the surface and at infinity $\theta_{z 0}$ and $\theta_{z^{\infty}}$, the radiative-equilibrium height scale $H_{R}$, the forcing time scale $\tau$, and the rotation parameters $f_{0}$ and $\beta$.

The tropopause height is estimated from the isentropic mass circulation as described in section 2, with the isentropic diagnostics averaged over "baroclinic zones." These are defined as the regions over which the vertically integrated eddy meridional heat flux is within a $25 \%$ of its latitudinal maximum. The radiative constraint and marginal criticality predictions are calculated using data diagnosed from the model's mean state, averaged over the same baroclinic zones. The idealized radiative constraint prediction is based on the equal-area construction of Fig. 3 using the simulated stratification $\partial_{z} \bar{\theta}$, while the marginal criticality prediction (Charney height $)$ is given by $h=-(f / \beta) \partial_{y} \bar{\theta} / \partial_{z} \bar{\theta}$, with the Coriolis parameter evaluated at the latitude of maximum meridional heat flux and the temperature gradients obtained from the simulations. With both predictions, there is some ambiguity depending on the level at which temperature gradients are evaluated; we show results using both midtropospheric values and tropospheric averages. We also tested the marginal criticality constraint using values at the surface and at the top of the boundary layer, but the agreement was worse than with the previous conventions.

As discussed in the previous section, we expect the idealized radiative constraint to work better when the dynamical adjustment in stratification is significant and the tightness approaches unity. In that limit the radiative constraint also tends to be flat $\left(s_{H}\right.$ is small) and the troposphere is not allowed to expand freely. Figures $4 \mathrm{~b}$ and $4 \mathrm{c}$ show that the main external parameter affecting these properties is the $\theta_{z 0} / \theta_{z \infty}$ ratio, with the tightness increasing and the steepness decreasing as this ratio becomes more negative. We thus start by studying the behavior of our model when $\theta_{z 0}$ is varied, keeping $\theta_{z^{\infty}}$ constant. These simulations are mapped in Figs. $4 \mathrm{~b}$ and $4 \mathrm{c}$, using the mean tropospheric stratification (diagnosed from the model) as an estimate for the "adjusted" stratification $\widetilde{\theta}_{z}$. The specific values of $T$ and $s_{H}$ covered are shown in Fig. 4d.

Figure 6 shows some diagnostics for these simulations. We can see that the tropopause rises when $\theta_{z 0}$ decreases, as predicted by the idealized radiative constraint. With small (negative) $\theta_{z 0}$ the two radiative-constraint predictions are in good agreement with each other and with

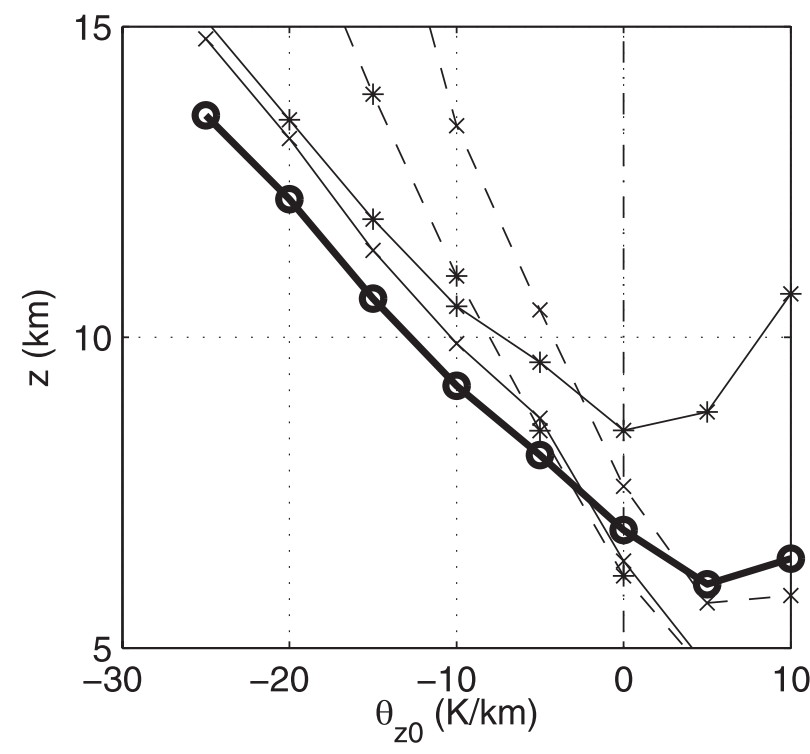

FIG. 6. Simulated tropopause height (thick solid) and predictions based on the radiative constraint (thin solid) and marginal criticality (dashed) for runs varying the surface radiative-equilibrium stratification $\theta_{z 0}$. Two sets of predictions are shown using tropospheric averages $(*)$ or midtropospheric levels $(\times)$, based on the simulated tropopause height.

the observed tropopause height. However, as $\theta_{z 0}$ increases, the two predictions diverge and they also become poor estimates of the actual tropopause. The idealized radiative constraint tends to do better than marginal criticality (with both conventions) for small $\theta_{z 0}$, when this construction is most reliable, but both predictions display comparable skill around the control value $\theta_{z 0}=0$. For larger values of $\theta_{z 0}$ the radiative constraint becomes loose and marginal criticality appears to perform better. This suggests that the radiative constraint may be preventing the adjustment to marginal criticality when this constraint is tight. Given the importance of the $\theta_{z 0}$ parameter, we have grouped our simulations in 4 different sets using the following values of $\theta_{z 0}:-20,-10,0$, and $5 \mathrm{~K} \mathrm{~km}^{-1}$. For each of these sets, variations in the remaining parameters are considered. Overall, 117 experiments were performed; these are listed in Table 1.

Figure 7 describes the sensitivity of tropopause height to external parameters for the set with $\theta_{z 0}=$ $-20 \mathrm{~K} \mathrm{~km}^{-1}$ (right) and for the control set with $\theta_{z 0}=0$ (left), which are representative of all our results. We can see that the tropopause rises with increasing $\delta_{Y}$ and $H_{R}$. When $f_{0}$ increases the tropopause rises with a weak dependence for the control set, but it remains roughly constant for the set with $\theta_{z 0}=-20 \mathrm{~K} \mathrm{~km}^{-1}$. The tropopause height also remains nearly constant against changes in $\beta$ for both sets and has weak variations (with different sign) in both sets when changing $\tau$. The larger 
TABLE 1. List of all simulations performed. For each set of runs (characterized by their $\theta_{z 0}$ value) we provide the total number of experiments performed in parentheses and the specific parameter variations considered in columns (only one parameter is changed at a time, with all other parameters kept at their control values). Parameter variations are expressed either as a range $p_{\min }: \Delta p: p_{\max }$ or as a list of values $p_{1}, p_{2}, \ldots, p_{n}$ when variations are not equally spaced.

\begin{tabular}{rlrrrr}
\hline \hline Set $(N)$ & $\delta_{y} / 2(\mathrm{~K})$ & \multicolumn{1}{c}{$\tau($ days $)$} & $H_{R}(\mathrm{~km})$ & $f_{0}\left(\times 10^{-4} \mathrm{~s}^{-1}\right)$ & $\beta\left(\times 10^{-11} \mathrm{~m}^{-1} \mathrm{~s}^{-1}\right)$ \\
\hline$-20(29)$ & $20: 10: 60$ & $1,2,5,10,20,40,80$ & $5: 5: 15$ & $1,1.5,2,2.5,3,4,5$ & $0.8: 0.4: 3.2$ \\
$-10(29)$ & $20: 10: 60$ & $2,5,10,20,40,80$ & $5: 5: 20$ & $1,1.5,2,2.5,3,4,5$ & $0.8: 0.4: 3.2$ \\
$0(32)$ & $20: 5: 55$ & $2,5,10,20,40,80$ & $5: 5: 20$ & $1,1.5,2,2.5,3,4,5$ & $0.8: 0.4: 3.2$ \\
$5(27)$ & $30: 10: 60$ & $5,10,20,40,80$ & $5: 5: 20$ & $1,1.5,2,2.5,3,4,5$ & $0.8: 0.4: 3.2$ \\
\hline
\end{tabular}

changes observed in tropopause height when changing $\delta_{Y}$ and $f_{0}$ for the control set compared to the $\theta_{z 0}=-20$ set are consistent with the larger values of $s_{H}$ over this part of the parameter space (cf. Fig. 4b). We next investigate whether the simulated sensitivity of tropopause height can be captured by the theoretical estimates.

\section{The two constraints for tropopause height}

Figure 7 also evaluates the adequacy of the radiative and marginal criticality constraints as predictors of changes in tropopause height when the parameters are varied. The idealized radiative constraint slightly underestimates the changes in tropopause height when varying $f_{0}$ with $\theta_{z 0}=0$, and also fails to predict the weak changes in $H$ when varying the diabatic time scale at large $\tau$. But other than that, it does really well for both sets, much better than we might have anticipated for the control set based on Fig. 5d. Even for the set of simulations with $\theta_{z 0}=$ $5 \mathrm{~K} \mathrm{~km}^{-1}$ (not shown) the idealized radiative constraint performs surprisingly well.

Marginal criticality also gives useful predictions for the control set, giving a sensitivity of the correct sign for tropopause height except when changing the diabatic time scale in the small $\tau$ limit. Nevertheless, this constraint (slightly) underestimates the changes in tropopause height when increasing $\delta_{Y}$ (Fig. 7a) and significantly overestimates the simulated tropopause rise when $f_{0}$ is increased (Fig. 7d). The former implies a (weak) reduction in criticality and the latter a criticality increase. But despite these shortcomings, there exists overall a remarkable tendency in the model's mean state to adjust to marginal criticality for this set.

In contrast, the set with $\theta_{z 0}=-20 \mathrm{~K} \mathrm{~km}^{-1}$ is characterized by large changes in criticality. The Charney height drops abruptly when increasing $\delta_{Y}, \tau$, and $\beta$ (Figs. 7f,g,j), yet the tropopause rises in the first case and remains roughly constant in the last two. In all cases, this implies a decrease in criticality. On the other hand, the Charney height increases steeply with $f_{0}$ (Fig. 7i) and becomes very large (out of the range displayed in the plot), despite the simulated tendency for the tropopause height to remain constant against changes in rotation. In summary, the criticality increases when $f_{0}$ increases and when $\delta_{Y}, \tau$, and $\beta$ decrease, with a sensitivity that is strongly modulated by the value of $\theta_{z 0}$ : the criticality changes are much larger with $\theta_{z 0}=-20 \mathrm{~K} \mathrm{~km}^{-1}$ than with $\theta_{z 0}=0$.

To summarize these results, Fig. 8 displays scatterplots of simulated tropopause height against the different predictors for the two sets discussed above, and we can see that the idealized radiative constraint has quantitative skill in many cases. The marginal criticality estimate has less predictive skill overall, but it does have some, especially for the $\theta_{z 0}=0$ set. To be more quantitative, Table 2 lists correlations between changes in the predictors and in tropopause height for all four sets, as well as correlations between the two radiative constraint predictions (using midtropospheric values or vertical averages) and the two marginal criticality predictions with each other. The two marginal criticality predictions correlate very well with each other in all cases, suggesting that changes in the vertical structure are of little importance. The correlation between the two radiative constraint predictions is also very high for low $\theta_{z 0}$ but drops significantly as the radiative constraint becomes loose. The predictive power of this constraint decreases over the same range, with the correlation between tropopause height and predictor dropping from a high value of 0.97 for $\theta_{z 0}=-20 \mathrm{~K} \mathrm{~km}^{-1}$ to a low value of 0.61 for $\theta_{z 0}=5 \mathrm{~K} \mathrm{~km}^{-1}$. In contrast, the marginal criticality predictions are essentially uncorrelated with tropopause height for small $\theta_{z 0}$ but their predictive skill is comparable to that of the idealized radiative constraint for the largest values of $\theta_{z 0}$.

The fact that marginal criticality works better when the radiative constraint becomes loose supports the argument given in the introduction that it is this constraint that prevents the adjustment to marginal criticality by preventing the tropopause from reaching as high as the Charney height, and that enables the flow to be supercritical. This is most transparent in Fig. 6, which shows that the idealized radiative constraint tightly determines the tropopause height when it predicts a lower height than marginal criticality, while it becomes loose precisely over the parameter range in which both constraints predict comparable heights. This may be interpreted as follows. The idealized radiative constraint tropopause 

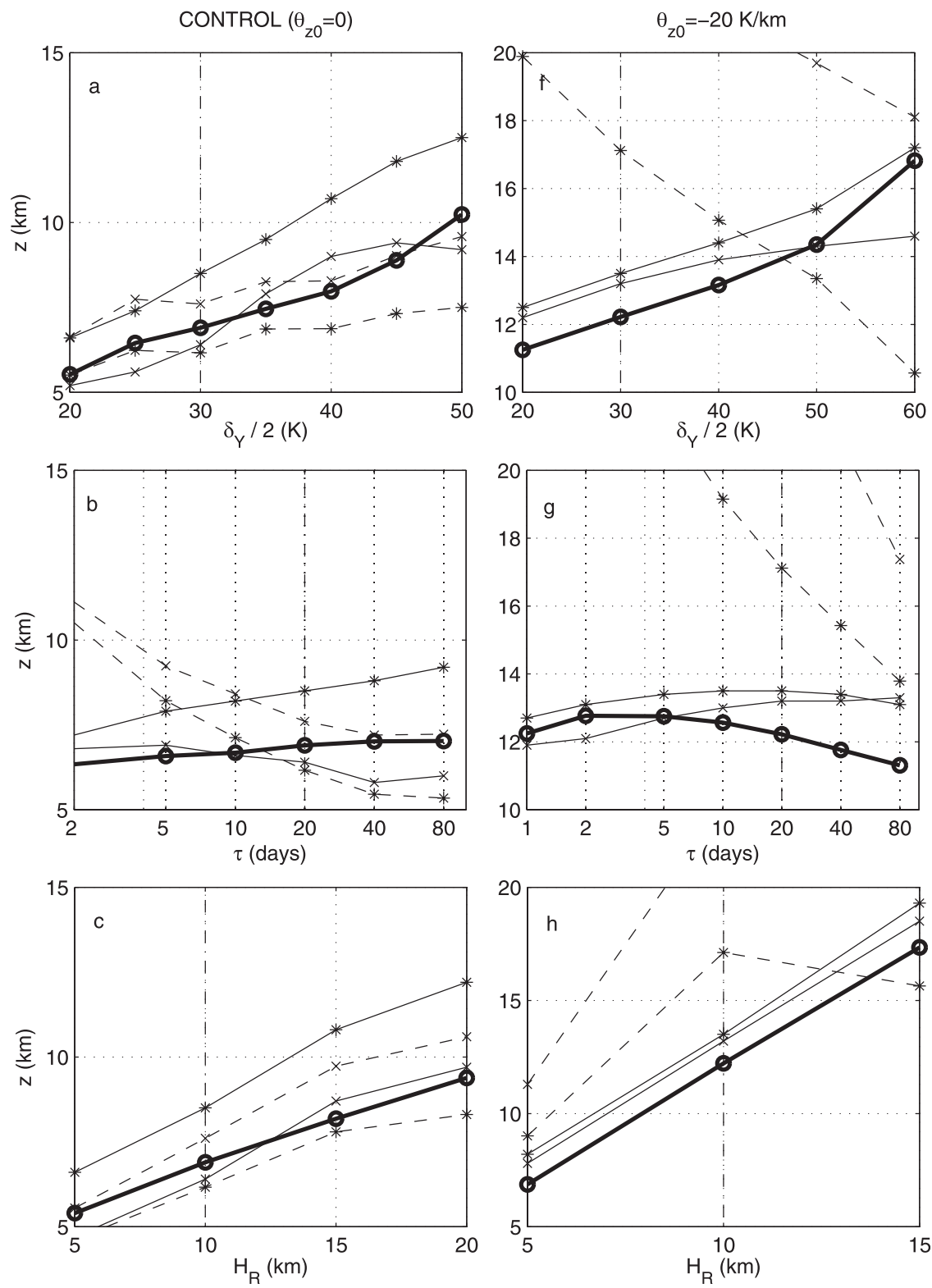

FIG. 7. As in Fig. 6, but varying the radiative-equilibrium baroclinicity $\delta_{Y}(\mathrm{a})$,(f); the forcing time scale $\tau$ (note the logarithmic scale) (b),(g); the radiative scale height $H_{R}(\mathrm{c}),(\mathrm{h})$; the mean Coriolis parameter $f_{0}(\mathrm{~d}),(\mathrm{i})$; and its derivative $\beta$ (e),(h). Panels correspond to runs with (a)-(e) the control value $\theta_{z 0}=0$ and (f) $-(\mathrm{j})$ convectively unstable radiative-equilibrium stratification $\left(\theta_{z \infty}=-20 \mathrm{~K} \mathrm{~km}^{-1}\right)$.

$H_{\text {rad }}$ represents the depth of the required mixing to have energy conservation with uniform stratification, but there is no reason why the mixing should necessarily extend that high if the dynamics dictate otherwise. In particular, if the Charney height were lower than this depth one would expect to observe the most unstable mode mixing over that (lower) height, with approximate radiative equilibrium aloft. To be sure, the radiative constraint (energy conservation) must still be satisfied in that case, but this can only be compatible with radiative equilibrium below $H_{\mathrm{rad}}$ in the presence of nonuniform stratification. In other words, it is not the radiative constraint that fails, only the idealized construction of Fig. 3 that assumes a uniform stratification. We refer to this as a loose radiative constraint.

This suggests that if we were to set $\theta_{z 0}=\theta_{z^{\infty}}$, as in the pure Charney-Boussinesq problem, nothing would prevent the flow from adjusting to marginal criticality, since 

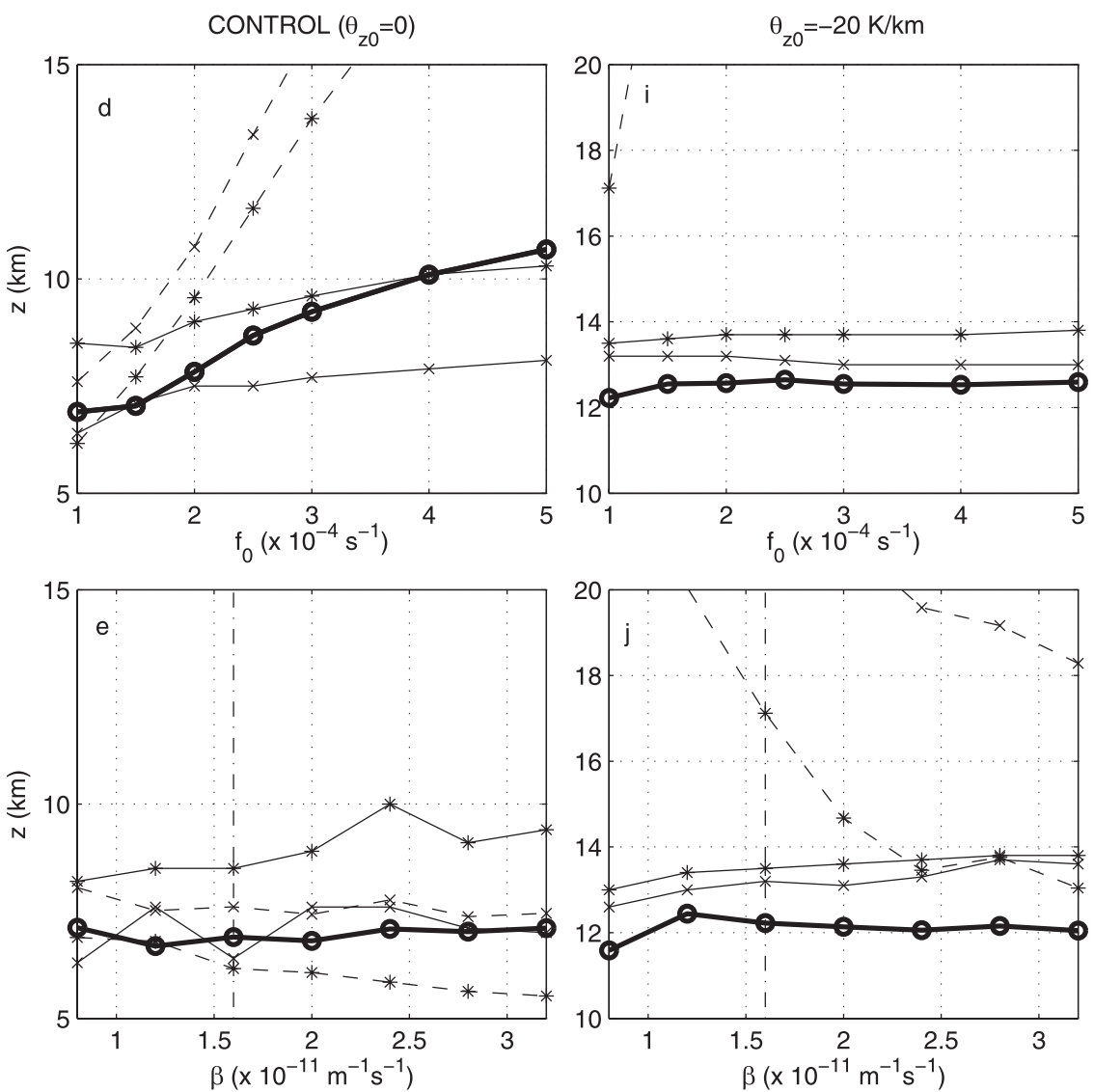

FIG. 7. (Continued)

that would be essentially equivalent to taking $H_{\text {rad }} \rightarrow \infty$. In that limit we would expect the depth of the mixing to be given by the Charney height, with approximate radiative equilibrium above that height. The radiative constraint (energy conservation) would still need to be satisfied, of course, but note that only deviations in the stratification from its mean value would play a role in the equal-area construction. In other words, the verticalmean stratification is essentially unconstrained by energy conservation in the Charney limit. Simulations performed in this setting support our speculation about an adjustment to marginal criticality in that limit (not shown), although these simulations are somewhat ambiguous because they tend to have a less sharp tropopause and because it is harder to constrain the vertical scale of the eddies with a uniform stratification, making the influence of the upper computational boundary more evident in these runs.

\section{Summary and conclusions}

We shall state our conclusions compactly in this opening paragraph and then expand them in the rest of this section.
The tropopause height may be considered to be the outcome of two constraints, one dynamical and one radiative. In this paper we have investigated the degree to which an idealized expression of the radiative constraint and a particular dynamical constraint, the assumption of marginal criticality, provide useful predictions of the tropopause height. (In its most general form the radiative constraint must always be satisfied, but it need not necessarily provide a tight constraint.) We find that both constructions have some predictive value but, depending on the diabatic forcing, neither is always useful. Depending on the form of the diabatic forcing, the radiative constraint may be loose and not provide an effective constraint on the tropopause height, or it may be tight and constrain the tropopause to a height lower than the Charney height, leading to supercritical flow.

Marginal criticality is a state in which the tropopause height scales with the Charney height, which in turn represents the depth over which the integrated positive potential vorticity (PV) gradient in the interior is comparable to the (integrated) negative delta function at the surface (Zurita and Lindzen 2001). Modes with this vertical scale thus tend to have a PV diffusivity with 

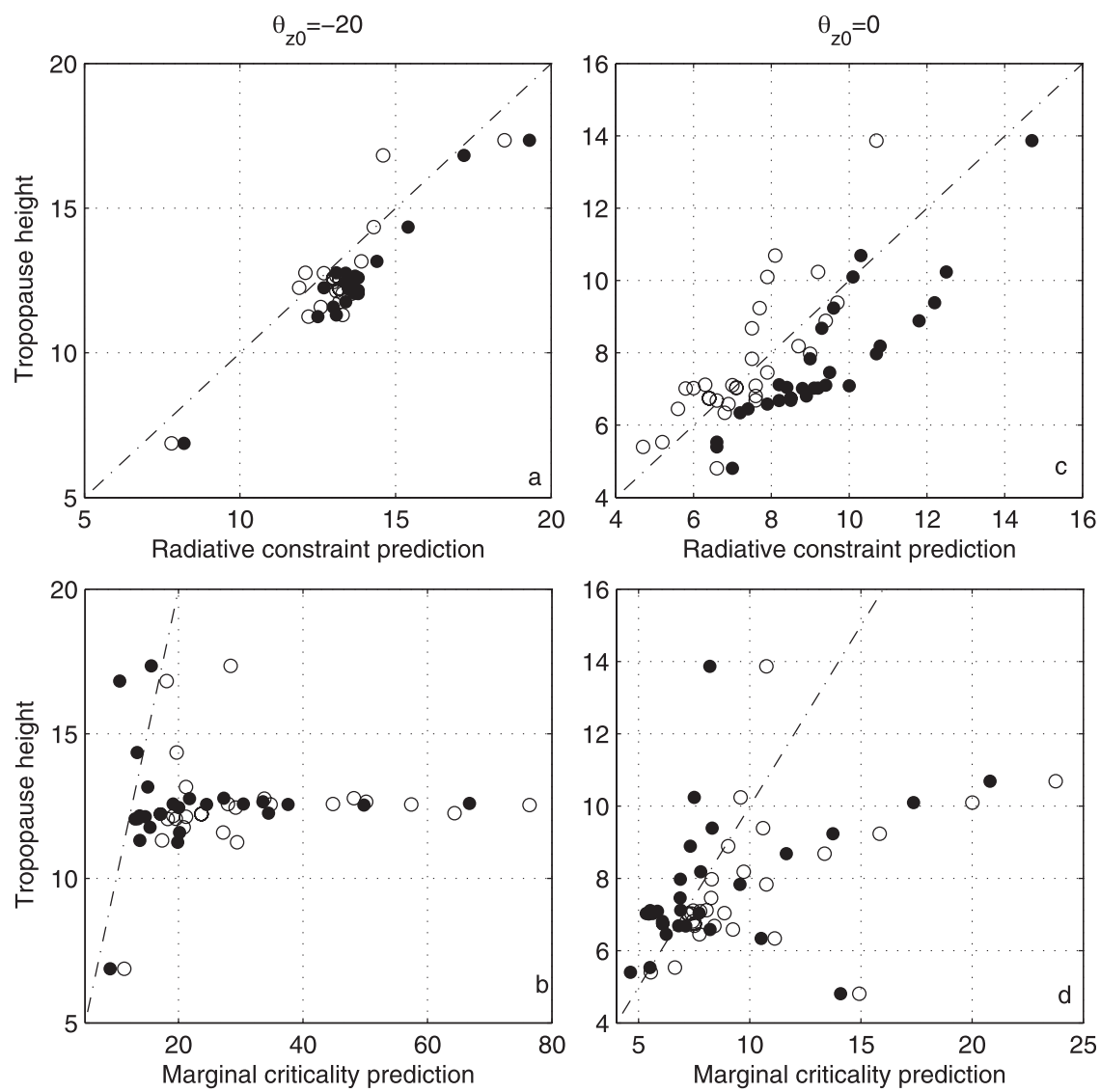

FIG. 8. For the set with $\theta_{z 0}=-20 \mathrm{~K} \mathrm{~km}^{-1}$, scatterplots displaying the correlation between tropopause height and (a) the radiative constraint using the vertically integrated (filled) or midtropospheric (empty) stratification; and (b) the marginal criticality constraint based on vertically integrated (filled) or midtropospheric (empty) values. (c),(d) As in (a),(b), but with $\theta_{z 0}=0$.

broad vertical structure and comparable values at the surface and in the interior because the eddy PV flux must integrate vertically to zero (when eddy momentum fluxes are neglected). Since this represents an optimal structure for extracting energy from the mean flow, the most unstable mode should scale with that height and this is the structure that we might expect to emerge in an unforced, adiabatic flow.

However, while we would expect the eddies to extend to this height in the unforced, adiabatic case, the same need not be true in the presence of a radiative constraint that essentially limits the excursions of tropopause height. That could certainly be possible, if the flow rearranges the mean temperature structure meridionally and vertically in such a way that the Charney height agrees with the tropopause height predicted by the radiative constraint. The main objection to this idea is that this would imply that the meridional eddy PV fluxes (or the implied diffusivity) cannot be just a function of the mean state, but must also be an explicit function of the heating. This

TABLE 2. Correlations between tropopause height $(H)$ and predictions based on the radiative constraint (RC) and marginal criticality (MC) when varying the external parameters for all sets of runs, using different conventions for each estimate (VI: vertically integrated; MT: midtroposphere). We also show correlations between the estimates under both conventions, indicative of the robustness against changes in vertical structure.

\begin{tabular}{rcccccc}
\hline \hline Set & $H, \mathrm{RC}_{\mathrm{VI}}$ & $H, \mathrm{RC}_{\mathrm{MT}}$ & $H, \mathrm{MC}_{\mathrm{VI}}$ & $H, \mathrm{MC}_{\mathrm{MT}}$ & $\mathrm{MC}_{\mathrm{VI}}, \mathrm{MC}_{\mathrm{MT}}$ & $\mathrm{RC}_{\mathrm{VI}}, \mathrm{RC}_{\mathrm{MT}}$ \\
\hline-20 & 0.97 & 0.89 & 0.03 & 0.08 & 0.99 & 0.96 \\
-10 & 0.96 & 0.89 & 0.19 & 0.27 & 0.99 & 0.96 \\
0 & 0.89 & 0.82 & 0.56 & 0.63 & 0.99 & 0.92 \\
5 & 0.61 & 0.72 & 0.83 & 0.87 & 0.58 \\
\hline
\end{tabular}


would be unusual and is not supported by previous studies in models with rigid lids, in which the eddy fluxes vary smoothly with the mean state (Zurita-Gotor and Vallis 2009). Nevertheless, some studies have reported a robust adjustment to marginal criticality (e.g., Schneider and Walker 2006), which seems consistent with observations that show that the isentropic slope does not vary as much with season as either the horizontal or vertical gradients do separately (Stone and Boaz 1996).

To better understand the interaction of the radiative and dynamical constraints, we used a primitive equation model with a simple Newtonian heating formulation in which we can, to some degree, control the effectiveness of the radiative constraint. This constraint essentially reflects conservation of energy when heat is redistributed vertically by the dynamical fluxes. This leads to a relation between tropopause height and stratification under the additional assumptions that the stratification is uniform and the stratosphere is in strict radiative equilibrium. Although these assumptions are never exactly satisfied (and in principle the former could be relaxed, if one had a theory for the vertical structure of the stratification), they are a useful approximation when the dynamical vertical heat transport is large since the energy balance then is only minimally affected by the violation of these assumptions. Thus, the idealized radiative constraint with uniform stratification will represent a strong constraint on the system when the vertical redistribution of heat is important and a weak constraint when it is small, or potentially if the diabatic forcing has special forms.

To quantify the effectiveness of the radiative constraint, we have defined a tightness parameter based on the dynamical adjustment in stratification at tropopause levels. When the radiative constraint is loose, the idealized constraint is only weakly satisfied and the tropopause height is free to adjust. If the radiative constraint predicts tropopause heights on the order of or higher than the Charney height, then we find that the flow does tend to adjust to marginal criticality. In contrast, the radiative constraint prevents the expansion of the eddies when it predicts tropopause heights lower than the Charney height, giving rise to supercriticality. That is to say, if the thermodynamic forcing is chosen to be such that vertical transports are large (or of course if the thermodynamic forcing directly produces a rigid lid below the Charney height), then supercritical flow can arise. In such cases determining the dynamical constraint becomes, effectively, the same as constructing a theory for the lateral eddy fluxes in the continuously stratified, fully nonlinear case, for which one requires a theory of the transport properties of geostrophic turbulence.

Put simply, our results suggest that in some circumstances the radiative constraint will prevent the adjustment to marginal criticality, so that this adjustment can only occur when the radiative constraint becomes loose or otherwise ineffective. That is, the particular form of the heating plays a role in determining the supercriticality of the flow, and a useful measure of this is the tightness of the radiative constraint. This may explain why results with comprehensive GCMs find that radiative constraint is useful but that supercritical flow may also arise. To test this hypothesis further, and to explore the relevance of this point of view to more complex models and to the real atmosphere, we are currently investigating the sensitivity of a model forced using gray radiation, which has a very different radiative constraint than Newtonian relaxation.

Acknowledgments. P. Z-G is supported by the Ministerio de Ciencia e Innovación of Spain under a Ramón y Cajal position, and by the MOVAC (Grant 200800050084028 from the Ministerio de Medio Ambiente, y Medio Rural y Marino of Spain) and DEVIAJE (CGL2009-06944) projects. GKV acknowledges support from NSF (OCE-1027603) and from the DoE.

\section{REFERENCES}

Birner, T., 2010: Residual circulation and tropopause structure. J. Atmos. Sci., 67, 2582-2600.

Branscome, L. E., 1983: The Charney baroclinic stability problem: Approximate solutions and modal structures. J. Atmos. Sci., 40, 1393-1409.

Frierson, D. M. W., I. M. Held, and P. Zurita-Gotor, 2006: A grayradiation aquaplanet moist GCM. Part I: Static stability and eddy scale. J. Atmos. Sci., 63, 2548-2566.

Green, J. S. A., 1970: Transfer properties of the large-scale eddies and the general circulation of the atmosphere. Quart. J. Roy. Meteor. Soc., 96, 157-185.

Held, I. M., 1978: The vertical scale of an unstable baroclinic wave and its importance for eddy heat flux parameterizations. J. Atmos. Sci., 35, 572-576.

, 1982: On the height of the tropopause and the static stability of the troposphere. J. Atmos. Sci., 39, 412-417.

-, and M. J. Suarez, 1994: A proposal for the intercomparison of the dynamical cores of atmospheric general circulation models. Bull. Amer. Meteor. Soc., 75, 1825-1830.

- and V. Larichev, 1996: A scaling theory for horizontally homogeneous, baroclinically unstable flow on a beta plane. J. Atmos. Sci., 53, 946-952.

— , and T. Schneider, 1999: The surface branch of the zonally averaged mass transport circulation in the troposphere. J. Atmos. Sci., 56, 1688-1697.

Lewis, R., Ed., 1991: Meteorological Glossary. 6th ed. Her Majesty's Stationery Office, $335 \mathrm{pp}$.

Lindzen, R. S., 1993: Baroclinic neutrality and the tropopause. J. Atmos. Sci., 50, 1148-1151.

Marshall, J., A. Adcroft, C. Hill, L. Perelman, and C. Heisey, 1997: A finite-volume, incompressible Navier-Stokes model for studies of the ocean on parallel computers. J. Geophys. Res., 102, 5753-5766.

Schneider, T., 2004: The tropopause and the thermal stratification in the extratropics of a dry atmosphere. J. Atmos. Sci., 61, $1317-1340$ 
and C. C. Walker, 2006: Self-organization of atmospheric macroturbulence into critical states of weak nonlinear eddyeddy interactions. J. Atmos. Sci., 63, 1569-1586.

— stratification of the extratropical troposphere. J. Atmos. Sci., 65, 3571-3583.

Stone, P. H., 1972: A simplified radiative-dynamical model for the static stability of rotating atmospheres. J. Atmos. Sci., 29, 405-418.

1978: Baroclinic adjustment. J. Atmos. Sci., 35, 561-571.

, and N. Boaz, 1996: Baroclinic adjustment: A comparison between theory, observations, and models. J. Atmos. Sci., 53, $1663-1674$

Thuburn, J., and G. C. Craig, 1997: GCM tests of theories for the height of the tropopause. J. Atmos. Sci., 54, 869-882.

, and - 2000: Stratospheric influence on tropopause height: The radiative constraint. J. Atmos. Sci., 57, 17-28.
Vallis, G. K., 2006: Atmospheric and Oceanic Fluid Dynamics. Cambridge University Press, 745 pp.

WMO, 1957: Meteorology: A three-dimensional science. WMO Bull., 4, 134-138.

Zurita, P., and R. S. Lindzen, 2001: The equilibration of short Charney waves: Implications for PV homogenization in the extratropical troposphere. J. Atmos. Sci., 58, 3443-3462.

Zurita-Gotor, P., 2007: The relation between baroclinic adjustment and turbulent diffusion in the two-layer model. J. Atmos. Sci., 64, 1284-1300.

2008: The sensitivity of the isentropic slope in a primitive equation dry model. J. Atmos. Sci., 65, 43-65.

— , and G. K. Vallis, 2009: Equilibration of baroclinic turbulence in primitive equations and quasigeostrophic models. J. Atmos. Sci., 66, 837-863.

— and - 2010: Circulation sensitivity to heating in a simple model of baroclinic turbulence. J. Atmos. Sci., 67, 1543-1558. 
Copyright of Journal of the Atmospheric Sciences is the property of American Meteorological Society and its content may not be copied or emailed to multiple sites or posted to a listserv without the copyright holder's express written permission. However, users may print, download, or email articles for individual use. 